\title{
Política pública de transplante de órgãos no Brasil
}

\author{
Public organ transplantation policy in Brazil
}

Política pública de trasplante de órganos in Brasil

Dalria Lima de Souza Moreira ${ }^{1 *}$, Marcia Rosa Bresinski ${ }^{1}$, Carla Aparecida Tosta Canzian ${ }^{1}$, Geudicéia Ribeiro', Gabriela Vieira de Oliveira Piovezan¹, Marcus Antonius da Costa Nunes².

\section{RESUMO}

Objetivo: Analisar as políticas públicas de transplante implantadas no Brasil, identificando as estratégias que versem sobre o aumento no índice de doadores e os fatores que impedem a doação no Brasil. Revisão Bibliográfica: A política de transplante de órgãos é um avanço considerável em termo de medicina, vez que é uma alternativa terapêutica eficiente, constituindo em muitos casos na última alternativa de melhora ou cura, no tratamento de doenças crônicas incuráveis, constituindo assim, uma melhoria na qualidade de vida do indivíduo, submetido ao transplante. Considerações Finais: O Brasil por meio de políticas públicas, instituiu normativas visando estabelecer o procedimento de doação de órgãos, como o Sistema Nacional de Transplante e a Comissão Intra-Hospitalar de Doação de Órgãos e Tecidos para Transplante, que contribuiu para o aumento no índice de doadores. No entanto, o processo de doação de órgãos ainda é pouco compreendido pela sociedade e pelos profissionais da saúde, em que se constata a necessidade de melhor promover a conscientização através de programas e campanhas educacionais, visto que existem fatores que impedem o aumento no índice de doadores, como a recusa familiar, a crença na comercialização e a ausência de conhecimento.

Palavras-chave: Doação de órgãos, Transplante, Política pública.

\begin{abstract}
Objective: To analyze the public transplantation policies implemented in Brazil, identifying the strategies that deal with the increase in the donor rate and the factors that prevent donation in Brazil. Bibliographic Review: The organ transplant policy is a considerable advance in terms of medicine, since it is an efficient therapeutic alternative, constituting in many cases the last alternative of improvement or cure, in the treatment of incurable chronic diseases, thus constituting an improvement on the individual's quality of life, submitted to transplantation. Final Considerations: Brazil, through public policies, has instituted regulations aimed at establishing the organ donation procedure, such as the National Transplant System and the Intra-Hospital Commission for Donating Organs and Tissues for Transplant, which contributed to the increase in the index donors. However, the organ donation process is still poorly understood by society and health professionals, in which there is a need to better promote awareness through educational programs and campaigns, since there are factors that prevent the increase in the rate of donors, such as family refusal, belief in commercialization and lack of knowledge.
\end{abstract}

Keywords: Organ donation, Transplant, Public policy.

1 Tecnologia e Educação na Faculdade Vale do Cricaré, São Mateus - ES.

2 Universidade Federal de Santa Catarina (UFSC), Santa Catarina - SC.

*E-mail: mydalrialima@hotmail.com. 


\section{RESUMEN}

Objetivo: Analizar las políticas públicas de trasplante implementadas en Brasil, identificando las estrategias que abordan el aumento de la tasa de donantes y los factores que impiden la donación en Brasil. Revisión Bibliográfica: La política de trasplante de órganos es un avance considerable en materia de medicina, ya que es una alternativa terapéutica eficaz, constituyendo en muchos casos la última alternativa de mejora o cura, en el tratamiento de enfermedades crónicas incurables, constituyendo así una mejora. sobre la calidad de vida del individuo sometido a trasplante. Consideraciones Finales: Brasil, a través de políticas públicas, ha instituido regulaciones destinadas a establecer el procedimiento de donación de órganos, como el Sistema Nacional de Trasplantes y la Comisión Intrahospitalaria de Donación de Órganos y Tejidos para Trasplante, que contribuyeron al aumento del índice. donantes. Sin embargo, el proceso de donación de órganos aún es poco conocido por la sociedad y los profesionales de la salud, en los que existe la necesidad de promover mejor la sensibilización a través de programas y campañas educativas, ya que existen factores que impiden el aumento de la tasa de donantes, como el rechazo familiar, la creencia en la comercialización y la falta de conocimiento.

Palabras clave: Donación de órgano, Trasplante, Política pública.

\section{INTRODUÇÃO}

O início do processo de transplante no Brasil, ocorreu no ano de 1964, através de um transplante renal, procedimento realizado no estado do Rio de Janeiro, no Hospital dos Servidores (GARCIA CD, et al., 2015). O país no ano de 2018, ficou em primeiro lugar no que tange ao programa público de transplante de órgãos do mundo, ofertado através do Sistema Único de Saúde (SUS), em que $96 \%$ dos procedimentos foram realizados na rede pública (SOARES LSS, et al., 2020).

No Brasil, instituiu-se o Sistema Nacional de Transplantes, formado por 27 Centrais Estaduais de Transplantes; 13 Câmaras Técnicas Nacionais; 504 estabelecimentos de Saúde e 851 serviços habilitados; 1.157 equipes de transplante; 574 Comissões intra-hospitalares de Doações e transplante e 72 Organizações de Procura de Órgãos (OPSs) (MINISTÉRIO DA SAÚDE, 2018).

O Ministério da Saúde (2018) ainda pontou aumento na taxa de transplantes no Brasil, que sucedeu no primeiro semestre de 2018 comparado ao mesmo período no ano de 2017. E esse crescimento contribui na vida dos pacientes portadores de doenças crônicas incuráveis, permitindo uma melhor condição de vida (PROGRAMA EINSTEIN DE TRANSPLANTES EM NÚMEROS, 2016). Victorino JP e Ventura CAA (2016) destacam que a doação de órgãos possibilita o retorno do paciente a vida diária, em que possibilita o retorno das atividades pessoais e laborais, e o aumento da sobrevida daquelas pessoas com doenças que comprometiam o funcionamento de órgãos específicos, no qual o transplante seria o último recurso disponível para o tratamento.

Ocorre que, mesmo sendo crescente o número de transplantes, a demanda de órgãos para transplante também é crescente, e a relação da quantidade de pacientes na listagem de espera e os órgãos disponíveis ainda é desproporcional, acarretando em muitos casos, no falecimento da individuo sem que consiga receber o órgão (WESTPHAI GA, et al., 2016). Robson NZ, et al. (2010) destaca que a política de transplante de órgão e tecidos no Brasil enfrenta inúmeras objeções quanto ao procedimento, resultando assim em resistência quanto a sua execução.

Desta forma, ao abordar quanto aos transplantes de órgão, tecidos e células no Brasil, consta-se que existem fatores a considerar, dentre os quais se destacam a redução na taxas de notificação de potencias doadores, que são atribuídas a contraindicações médicas, recusa da família, desejo de não ser doador por parte manifesto em vida, lentidão no diagnóstico de morte encefálica, crenças religiosas e culturais, e ausência de conhecimento e/ou informação sobre doação de órgãos (BARRETO BS, et al., 2016).

Assim, a partir de dados bibliográficos, este artigo irá abordar e apresentar dados importantes quanto a doação e o transplantes de órgão no Brasil, pontuando a relação da oferta e demanda, as ações adotadas pelo Estado quanto a regulamentação dos procedimentos e a atuação do poder público em possíveis estratégias e ações que versem sobre o aumento do índice de doadores. 


\section{REVISÃO BIBLIOGRÁFICA}

\section{Evolução da Legislação de Transplante de órgãos no Brasil}

O transplante é caracterizado como um processo cirúrgico no qual ocorre a troca de um órgão, tecido ou célula de uma pessoa que esteja precisando, por outro normal de um doador, que esteja vivo ou morto (MINISTÉRIO DA SAÚDE, 2008).

Existem três tipos de procedimento de transplantes: o autoplásico, heteroplásico e heterólogo. Sendo o transplante heterólogo, o processo no qual os tecidos ou órgãos são implantados em um organismo de outra espécie, já o transplante autoplásico é o procedimento em que se retira células ou tecidos de uma pessoa para a implantação em local diverso da mesma pessoa, e o heteroplásico é o procedimento em que capta células, tecidos ou órgãos de uma pessoa a fim de transplanta-lo em outro (FREITAS IO, 2018).

Assim, visando regular os procedimentos de transplante, o Brasil buscou normatizar política de doação de órgãos por meio de norma que estabelecesse os critérios, técnicas, responsabilidade, visando amparar os cidadãos que precisam de um órgão ou tecido para sobrevivência. Destacando o papel do Estado no fomento de ações que atendam aos interesses e necessidade da população, que no caso de políticas de transplantes, em que se assegura o direito à saúde, nos termos da Constituição Federal (BRASIL, 1988).

Desta forma, o início da legislação que abordava quanto ao transplante de órgãos e tecidos no Brasil, compreende a Lei $n^{\circ}$ 5.479/68, que regulamentou a retirada e transplante de tecidos, órgãos e partes de cadáver (BRASIL, 1968).

Sendo que somente em 1988, ocorreu de fato o aperfeiçoada a legislação sobre o tema, vez que a Constituição Brasileira, trouxe em seu artigo 199, inciso IV, as diretrizes relacionadas ao transplante em âmbito nacional, que dispunha não apenas sobre a retirada e transplante, mas também tratava do processo de coleta, processamento e transfusão de sangue, bem com quanto a proibição de qualquer tipo de comercialização de órgão e tecidos (BRASIL, 1988).

Anos depois, em 1992, foi publicada a Lei № 8.489/92, que trata quanto a retirada de transplantes de tecido, órgãos e partes do corpo humano, para finalidades terapêuticos e científicos, regulamentada pelo Decreto no 879/93 (BRASIL, 1992).

Não obstantes as normas já instituídas, ainda existia um impasse quanto ao transplante no Brasil, em razão do que ocorre hoje, a maioria dos transplantes são realizados de órgãos de pessoas mortas, por esta razão, em 1997, mediante a Resolução do Conselho Federal de Medicina ํㅡ 1.480/97, ocorreu a regulação do diagnóstico de Morte Encefálica (ME), sendo definida pela norma como o parada de todas as funções celebrais, incluindo tronco cerebral, o que resulta no processo irreversível (CONSELHO FEDERAL DE MEDICINA, 1997).

No mesmo ano, com o objetivo de promover melhorias no processo de transplante de órgãos, foi editada a Lei no 9.434/97, a chamada Lei dos Transplantes, com vigência nos dias atuais, a norma trata sobre a remoção de órgãos, tecidos com a finalidade de transplante e tratamento, e visou a regulamentação do processo de retirada de órgãos e todo o procedimento de transplantes no Brasil (BRASIL, 1997).

Sendo também público no mesmo ano o Decreto no 2.268/1997, que instituiu o Sistema Nacional de Transplante (SNT) e as instâncias em âmbito estadual e municipal, com a finalidade de aumentar o índice de órgãos doados (BRASIL,1997).

Destaca-se que o STN é o órgão responsável pela coordenação e regulação da captação, além da distribuição de órgãos em todo o território brasileiro, através do Centro de Transplantes instalados nos Estados, e possuía a competência no que se refere a conscientização pública quanto o transplante de órgão, evidenciando a relevância do processo de doação, visando o fomento a vida (FREIRE ILS, et al., 2014).

Com a Lei no 9.434/97, que se estabeleceu as condutas e diretrizes da doação para a realização de transplante, além de deliberar quanto as regras referentes ao processo de doação em vida e pós-morte e as penalidades impostas, em caso de infração ou descumprimento do preceito legal. 
Sobre esse aspecto, Bellingham JM, et al. (2011) afirma que os procedimentos de transplante podem ser realizados com doadores vivos e pós-morte, destacando que os órgãos sólidos e tecidos podem ser provenientes de doadores vivos, falecidos com morte encefálica ou de parada cardíaca.

Segundo o Ministério da Saúde (2017) é considerado doador vivo, qualquer pessoa, que possuí a intenção de doar, desde que não traga prejuízo a sua saúde, podendo doar um dos rins, parte do pulmão, parte do fígado e da medula óssea.

A Lei no 9.434/97, deliberou que os parentes de até quarto, ou cônjuge podem ser doadores, quanto as pessoas que não possuam vinculo consanguíneo, só poderão ser doadores mediante autorização judicial, sendo dispensada em relação à medula óssea.

O Ministério da Saúde (2017) considera como doador falecido, todo o paciente diagnosticado por morte encefálica, sendo em muitos casos vítimas de problemas cerebrais, tais como traumatismo craniano ou acidente vascular cerebral, em que os órgãos a serem doados, são encaminhados a pacientes que precisam e estão na lista única de doação, definida pela Central de Transplantes da Secretaria de Saúde de cada estado, e sobre o controle do STN.

Salienta-se que a Lei no 9.434/97, trouxe em seu texto original, a inovação quanto a doação de órgão, adotando o princípio da doação presumida, vez que até então a doação era consentida, em que o indivíduo deveria manifestar em vida que possuía intenção de doar órgão em caso de óbito, já em relação a doação presumida, os indivíduos em que não constasse em seus documentos de identificação civil, que não era doador, poderiam ter seus órgãos e tecidos designados a doação, após a morte encefálica, até mesmo com a recusa da Família (CALIXTO ACV, 2019).

Contudo, diante das polêmicas que envolvem o critério proposto pela Lei de Transplante, no ano de 2001, foi publicada a Lei oㅜ 10.211, que alterou os critérios de doação constantes na 9.434/97, afastando a possibilidade da doação presumida, concernindo à família o direito de decidir quanto a doação ou não de órgãos do ente falecido, após resultado de morte encefálica (BRASIL, 2001).

No ano de 2017, publicou-se o Decreto de o 9.175/17, revogando o decreto o 2.268/97, regulamentando a Lei dos Transplantes, além de incluir a autorização da realização de doação pelo companheiro, o que não era possível nas normas anteriores, além de regularizar e organizar do Sistema Nacional de Transplante, para que alinhado ao Ministério da Saúde, possa atuar no processo de captação, distribuição de órgãos, tecidos e partes do corpo humano. (BRASIL, 2017).

Ocorre que paira muito discursão no tocante ao diagnóstico de morte encefálica no Brasil, previamente regido pelo Conselho Federal de Medicina (CFM), mediante Resolução ํㅜ 1.480/97, por esta razão em 2017, o CFM editou a Resolução no 2.173 , em que modificou os critérios adotados quanto ao diagnóstico de morte

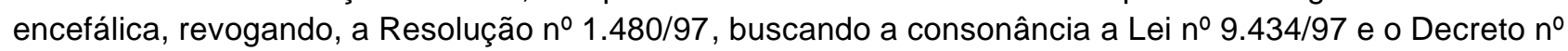
9.175/17 (BRASIL, 2017).

Além das normas mencionas, houve ainda a edição de portarias expedidas pelo Ministério da Saúde de no 1.752/05, que criou a Comissão Intra-hospitalar de Doação de Órgãos e Tecidos para Transplante em todos os estabelecimentos de saúde do país, e a de № 1.262/06, que aprovou o regulamento Técnico a fim estabelecer regras no que tange a potencialidade de doação de órgãos e tecidos nas Comissões Intrahospitalares de Doação de Órgãos e Tecidos para Transplante (CIHDOTT) (BRASIL, 2006).

Em 2009, foi publicado pelo Ministério da Saúde duas portarias, a de oㅡ 2.600, que aprovou o regulamento técnico do STN e a portaria no 2.601, que dispõe sobre o Plano Nacional de Implantação de Organizações de procura de órgão e tecidos (OPO), entretanto, mesmo diante da implantação deste plano nacional, no Brasil alguns estados desempenham a captação de órgãos por meio da Comissões Intrahospitalares de Doação de Órgãos e Tecidos para Transplante (BRASIL, 2009).

Desta forma, as políticas de normatização do Transplante de órgãos no Brasil, objetivam regulamentar e normatizar os procedimentos inerentes a transplante, mas ainda prescinde de aperfeiçoamento, visando assim, contribuir para o aumento do índice de doadores, vez que, o número de doadores ainda está aquém do número de pessoas constantes nas filas, a espera de um órgão. 


\section{A Política de notificação, captação e distribuição de órgãos e tecidos aplicada no Brasil}

Embora o Brasil possua um programa de doação de órgãos, sendo conhecido por ser o maior sistema público de transplantes de órgãos no âmbito mundial, ainda existem obstáculos que devem ser superados, vez que, a quantidade de doadores ainda é insuficiente em relação ao quantitativo de pessoas que necessitam de uma doação de órgão (VICTORINO JP e VENTURA CAA, 2016; SOARES LSS, et al., 2020).

Assim, a gestão nacional do SUS, através da Lei no 9.434/97, buscou estabelecer normas e diretrizes no que se refere ao procedimento de transplantes de órgãos, sendo posteriormente implantado o sistema centralizado de captação e distribuição de órgãos no país, por meio do Decreto em vigor de no 9.175/17. Deste modo, tem-se uma normativa que objetiva a regulamentação necessária quanto aos procedimentos de remoção e transplantes, a serem realizados por profissionais capacitados.

Existem dos dois tipos de transplantes, o que pode ocorrer entre intervimos, pouco aplicado, vez que somente é possível para alguns órgãos, a exemplo o rim, e o transplantes de doador falecido, em que somente é possível o transplante, quando o doador é diagnosticado por morte encefálica (SOARES LSS, et al., 2020).

Ocorrendo o diagnóstico de morte encefálica do potencial doador, cabe a Comissão Intra-hospitalares de Doação de Órgãos para Transplante organizar o processo de captação e remoção, notificando as Centrais Estaduais de Transplante, independente do consentimento da família, vez que tal procedimento é obrigatório a todos os estabelecimentos de saúde, sendo compromisso ético dos profissionais de saúde a realização de tal procedimento (MATTIA AL, et al., 2010).

Insta esclarecer que o diagnóstico de morte encefálica, segue um protocolo de verificação das condições do potencial doador, a confirmação do diagnóstico é realizada por dois médicos distintos através de exames clínicos, além de um exame complementar, conforme estabelecido pelo CFM pela resolução oㅡ 2.173/17 (BRASIL, 2017).

Nesse aspecto, a família do paciente é comunicada no momento da suspeita do diagnóstico de morte encefálica, sendo informados de todos os atos que serão realizados no paciente. E quando da confirmação, será realizada a entrevista familiar pela Comissão Intra-hospitalares de Doação de Órgãos e Tecidos para Transplante, que caso concorde, a comissão elabora o Termo de Autorização de Doação de Órgãos (MINISTÉRIO DA SAÚDE, 2017).

A Portaria de Consolidação no 04/17, do Ministério da Saúde, dispõe que o Termo de Autorização de Doação de Órgãos, deve ser encaminhado as Centrais Estaduais de Transplante para a realização do cadastro doador no STN, visando que a informação de disponibilidade de órgãos sejam disponibilizadas e seja feito o cruzamento das informações com os dados dos receptores cadastrados no Cadastro Técnico Único (MINISTÉRIO DA SAÚDE, 2017).

Os órgãos são distribuídos de acordo com as características clinicas e mediante a compatibilidade e gravidade dos pacientes receptores, de cordo com a logística e organização das equipes captadoras, sendo primeiramente ofertados em âmbito estadual, e não havendo receptor adequado e compatível, a oferta de estende a nível nacional, através do Sistema Nacional de Transplantes (CALIXTO ACV, 2019).

Sendo confirmado o receptor para o órgão disponibilizado, é realizada a cirurgia e feito o encaminhamento, levando em consideração o prazo de isquemia fria de cada órgão, vez que para cada órgão existe um prazo definido na Portaria de Consolidação no 04/17 (MINISTÉRIO DA SAÚDE, 2017).

\section{Dados dos transplantes de órgãos realizados no Brasil}

De acordo com dados constantes no Registro Brasileiro de Transplantes da Associação Brasileira de Transplantes de Órgãos (2019), em 2019 a taxa de doadores efetivos obteve um crescimento de 6,5\% no ano, atingindo $18,1 \mathrm{pmp}, 10,5 \%$, destacando que esse acréscimo, foi oriundo do aumento de $5,2 \%$ na taxa de potencias doadores em morte encefálica, mesmo assim, sendo considerado um ano difícil, com a recuperação da taxa de doadores e de transplante apenas no último trimestre, associada à campanha nacional de doação, que aconteceu em setembro, bem como a divulgação sobre a doação dos órgãos do apresentador Gugu Liberato. 
Ainda segundo o registro a taxa $(54,7 \mathrm{pmp})$ corresponde a $78 \%$ da taxa de morte encefálica prevista para o Brasil (70 pmp), devendo ser destacado, o aumento de 7,1\% na taxa de autorização familiar, que resultou em $60 \%$.

No ano de 2019, os transplantes de órgãos, exceto dos pulmonares, cresceram, em que o transplante renal ultrapassado os 6.000 (30 pmp) com aumento de $5,2 \%$, tanto nos casos de transplante com doador falecido $(5,5 \%)$ quanto com doador vivo $(4,1 \%)$, o transplante de córneas, que havia reduzido $11 \%$, de 2012 a 2018, estabilizou em 2019, com aumento de $0,6 \%$, e o transplante cardíaco, que já contava com crescimento constante desde 2011, aumentou 6\% no ano 2019, cerca de (2 pmp) (ASSOCIAÇÃO BRASILEIRA DE TRANSPLANTES DE ÓRGÃOS, 2019) (Tabela 1).

Tabela 1 - Necessidade estimada e número de transplantes realizados no Brasil em 2019.

\begin{tabular}{cccccc}
\hline \multirow{2}{*}{ Necessidade anual estimada } & \multicolumn{5}{c}{ Número de transplantes } \\
\cline { 2 - 5 } & Córnea & Rim & Fígado & Coração & Pulmão \\
\hline Necessidade estimada & 18.765 & 12.510 & 5.212 & 1.876 & 1.668 \\
Transplantes realizados & 14.943 & 6.283 & 2.245 & 380 & 106 \\
\hline
\end{tabular}

Fonte: Moreira DLS, et al., 2020. Dados extraídos de Registro Brasileiro de Transplantes, da Associação Brasileira de Transplantes de Órgãos.

No relatório, o Brasil é o segundo no ranking mundo em realizações de transplantes renais no ano de 2018, com a realização de 5.947 transplantes, o segundo também em transplantes hepáticos, com a realização de 2.195 procedimentos, sendo o número de doadores efetivos 17,0 pmp, e o número de transplantes com doadores falecidos 24,1 pmp.

\section{Fatores que dificultam ou impedem a concretização do Transplante no Brasil}

Calixto ACV (2019) destaca que o transplante é a última alternativa de melhoria ou de cura, no tratamento de várias doenças crônicas consideradas incuráveis, ou seja, sendo a única chance que um paciente no que se refere a expectativa de vida. Entretanto, mesmo como referência em transplantes, existem muitos fatores que dificultam o aumento no índice de doares.

Mesmo sendo um procedimento seguro, permitindo melhorias na condição de vida, muitas pessoas não possuem a intenção de doar órgãos e tecidos, e isto está associado a diversos fatores, tais como: baixas taxas de notificação de potenciais doares e de efetivação da doação, contradições medicas, recusa dos familiares, desejo de não doar doador em vida, morosidade no diagnóstico de morte encefálica, crenças religiosas, falta de informação e conhecimento sobre a doação de órgãos (SOARES LSS, et al., 2020).

Como fator, destaca-se a falta de desconfiança do sistema único de saúde, em que algumas famílias e profissionais da saúde associam diagnóstico de morte encefálica, com o objetivo de induzir a doação de órgãos, ou até mesmo ao comercio (ALMEIDA EC, et al., 2015).

Calixto ACV (2019) através de estudo, destaca que a crença na comercialização e fator que impede o aumento do índice de doadores de órgão é está presente na população, e até mesmo entre os profissionais de saúde não capacitados ou que não possuem conhecimento adequado sobre o procedimento. Outra causa apontada como recusa para doação de órgãos é a crença na promessa de ressureição (BOUSSO RS, et al., 2011). A baixa de índices de doação de órgão está associada a recusa familiar ligada, quanto a ausência de conhecimento e informação quanto ao diagnóstico de morte encefálica, pois associam a via a presença de batimentos cardíacos, todavia, o conceito de mortes encefálica compreende a perda completa e irreversível das funções encefálicas, em que é possível a constatação e comprovação de que as funções vitais, podem ser preservada, mesmo diante um quadro de ausência de atividade cerebral (FREIRE ILS, et al., 2014).

Nesse aspecto, dados do registro demostram que da maioria dos casos notificados, que correspondem aos potencias doadores, considerada as pessoas que estão no protocolo de morte encefálica, no ano de 2019 apenas $60 \%$ destes, tornaram-se doadores efetivos, e os outros $40 \%$ representam as famílias que não autorizaram a doação (Tabela 2). 
Tabela 2 - Registro de Doações de órgãos no Brasil, 2019.

\begin{tabular}{lrrc}
\hline Doações de órgãos & $\mathbf{2 0 1 7}$ & $\mathbf{2 0 1 8}$ & $\mathbf{2 0 1 9}$ \\
\hline Número de doadores efetivos & 3.415 & 3.534 & 3.768 \\
Número de doadores efetivos (pmp) & 16,6 & 17,0 & 18,1 \\
Número de notificações (potenciais doadores) & 10.629 & 10.782 & 11.399 \\
Número de notificações (pmp) & 51,6 & 51,9 & 54,7 \\
Recusa familiar & 2.740 & 2.753 & 2.674 \\
Percentual de recusa das entrevistas & $42 \%$ & $43 \%$ & $40 \%$ \\
Parada cardíaca & 1232 & 988 & 927 \\
Contraindicação médica & 1559 & 1.545 & 1.761 \\
Outros & 1683 & 1.961 & 2.269 \\
\hline
\end{tabular}

Fonte: Moreira DLS, et al., 2020. Dados extraídos de Registro Brasileiro de Transplantes, da Associação Brasileira de Transplantes de Órgãos.

De acordo com Freire ILS, et al. (2014) a recusa ocorre por falta de informação e conhecimento quanto a importância do processo consciente de doação de órgãos e tecidos. Registra ainda, como fatores de recusa a doação de órgãos, a ausência de informação sobre o processo de doação e transplante, sendo destacado em estudo realizado em Valparaíso-GO, em que cerca de $80 \%$ dos entrevistados apontaram a televisão como fonte de informação (FELIX MB e SANTOS WL, 2015).

\section{CONSIDERAÇÕES FINAIS}

O processo de doação de órgãos é uma forma de auxiliar na qualidade de vida de pessoas, e que mesmo sendo um procedimento devidamente normatizado, ainda é pouco compreendido pela sociedade e também pelos profissionais da saúde, em que evidencia-se a necessidade de que órgãos públicos promovam campanhas educacionais de que versem sobre a campanha de atenção à saúde, alertando quanto a importância de realizar a doação de órgãos, assim como explanando sobre o processo de doação de órgãos em todos os níveis de ensino. Destaca-se ainda a importância quanto a viabilização de campanhas que incentivem a doação nos meios de comunicação, com a finalidade de atingir o maior número de pessoas possível, buscando ampliar o conhecimento da sociedade quanto ao assunto e acabar com a desinformação e crenças referente ao processo de doação de órgãos, ainda existentes na população brasileira.

\section{REFERÊNCIAS}

1. ALMEIDA EC, et al. Atuação de profissionais de saúde em doação de órgãos na perspectiva do familiar: uma análise problematizadora. Arq. Cienc. Saúde UNIPAR, 2015; 19(2): 139-145.

2. ASSOCIAÇÃO BRASILEIRA DE TRANSPLANTE DE ÓRGÃO. Dimensionamento dos Transplantes no Brasil e em cada estado (2012-2019), 2019; 25(4): 1-88.

3. BARRETO BS, et al. Fatores relacionados à não doação de órgãos de potenciais doadores no estado de Sergipe, Brasil. Rev. Bras. Pesq. Saúde, 2016; 18(2):40-48.

4. BOUSSO RS, et al. Crenças religiosas, doença e morte: perspectiva da família na experiência de doença. Revista da Escola de Enfermagem, 2011; 45(2): 397-403.

5. BRASIL. Lei oㅜ 5.479, de 10 de agosto de 1968. Dispõe sobre a retirada e transplante de tecidos, órgãos e partes de cadáver para finalidade terapêutica e científica, e dá outras providências. Brasília (Brasil): Ministério da Saúde, 1968.

6. BRASIL. Constituição da República Federativa do Brasil, texto promulgado em 05 de outubro de 1988. Brasília (Brasil), 1988.

7. BRASIL. Resolução n. 2.173/2017. Define os critérios de diagnóstico de morte encefálica. Revoga a resolução 1.480/1997. Brasília (Brasil): Conselho Federal de Medicina, 2017.

8. BRASIL. Lei no 10.211 , de 23 de março de 2001. Altera dispositivos da Lei no 9.434, de 4 de fevereiro de 1997, que "dispõe sobre a remoção de órgãos, tecidos e partes do corpo humano para fins de transplante e tratamento". Brasília (Brasil), 2001.

9. BRASIL. Decreto № 2.268, de 30 de junho de 1997. Regulamenta a Lei oㅜ 9.434, de 4 de fevereiro de 1997, que dispõe sobre a remoção de órgãos, tecidos e partes do corpo humano para fim de transplante e tratamento, e dá outras providências. Brasília (Brasil), 1997.

10. BRASIL. Decreto $n^{\circ} 9.175$, de 18 de outubro de 2017. Regulamenta a Lei $n$ ㅇ 9.434 , de 4 de fevereiro de 1997 , para tratar da disposição de órgãos, tecidos, células e partes do corpo humano para fins de transplante e tratamento. Brasília (Brasil), 2017. 
11. BRASIL. Portaria de Consolidação no 4, de 28 de setembro de 2017. Consolidação das normas sobre os sistemas e os subsistemas do Sistema Único de Saúde. Ministério da Saúde. Brasília (Brasil), 2017.

12. CALIXTO ACV. Conhecimento de profissionais e trabalhadores da saúde sobre o processo de doação e transplante de órgãos e tecidos, GO. Dissertação (Mestrado em Enfermagem) - Universidade Federal do Goiás, Goiânia, 2019; $90 \mathrm{p}$.

13. FELIX MB, SANTOS WL. Conhecimento da população do município de Valparaíso de Goiás sobre o processo de doação de órgãos para transplantes. Revista de Divulgação Científica Sena Aires, 2015; 4(1): 3-10.

14. FREITAS IO. Morte Cerebral - Tipos de Transplantes. Terapia Intensiva - Práticas na Atuação da Enfermagem. São Paulo: Érica, 2018; 120 p.

15. FREIRE ILS, et al. Processo de doação de órgãos e tecidos para transplantes: reflexões sobre sua efetividade. Revista de Enfermagem UFPE On Line, 2014; 8(7): 2532-2539.

16. GARCIA CD, et al. Doação e transplante de órgãos e tecidos. São Paulo: Segmento Farma, 2015.; 560 p.

17. MATTIA AL, et al. Análise das dificuldades no processo de doação de órgãos: uma revisão integrativa da literatura. Revista Bioethicos, 2010; 4(1): 66-74. 2010.

18. MINISTÉRIO DA SAÚDE. Dicas em Saúde. Biblioteca Virtual em Saúde. Brasília (Brasil): Ministério da Saúde, 2008.

19. MINISTÉRIO DA SAÚDE. Brasil aumenta doação de órgãos e bate recorde em transplantes. Brasília (Brasil): Ministério da Saúde, 2018.

20. PROGRAMA EINSTEIN DE TRANSPLANTES EM NÚMEROS. 2016. In: Sociedade Beneficente Israelita Brasileira Albert Einstein. Disponível em: https://www.einstein.br/especialidades/transplantes/transplante-orgaos. Acesso em: 07 jul. 2020.

21. VICTORINO JP, VENTURA CAA. Bioética e Biodireito: da Doação ao Transplante de Órgãos. Brazilian Journal of Forensic Sciences, Medical Law and Bioethics, 2016; 6(1): 72-83.

22. WESTPHAL GA, et al. Diretrizes para avaliação e validação do potencial doador de órgãos em morte encefálica. Rev. Bras. Ter Intensiva, 2016; 28(3): 220-255. 